\title{
Conflit de civilisations : fondement théorique et significations pratiques. Partie 2
}

Wang Jisi

\section{(2) OpenEdition}

\section{Journals}

Édition électronique

URL : http://journals.openedition.org/conflits/894

DOI : $10.4000 /$ conflits.894

ISSN : $1777-5345$

Éditeur :

CCLS - Centre d'études sur les conflits lilberté et sécurité, L'Harmattan

Édition imprimée

Date de publication : 15 octobre 1995

ISSN : 1157-996X

\section{Référence électronique}

Wang Jisi, «Conflit de civilisations : fondement théorique et significations pratiques. Partie 2 »,

Cultures \& Conflits [En ligne], 19-20 | automne-hiver 1995, mis en ligne le 04 mars 2003, consulté le 30 mars 2021. URL : http://journals.openedition.org/conflits/894 ; DOl : https://doi.org/10.4000/conflits. 894

Ce document a été généré automatiquement le 30 mars 2021

Creative Commons License 


\section{Conflit de civilisations : fondement théorique et significations pratiques. Partie 2}

Wang Jisi

\section{UNE CONCEPTION REALISTE TRADITIONNELLE : A PROPOS DE LA LUTTE POUR LE POUVOIR}

1 Bien que Samuel Huntington mette clairement en évidence, dans sa théorie, le rôle du facteur spirituel, il ne nie jamais le rôle joué par la puissance. Dans les années 1987-1988, l'ouvrage de Paul Kennedy The Rise and Fall of the Great Powers a provoqué une controverse dans les milieux académiques américains. Principal représentant du courant "optimiste" au sein cette controverse, Huntington déclarait que les Etats-Unis n'étaient pas sur le déclin mais en pleine phase de prospérité et que l'attraction exercée par la démocratie politique et le libéralisme économique des Etats-Unis était plus forte que jamais ${ }^{1}$. Il semble qu'il soit soudainement devenu pessimiste. Constatant la réduction des différences économiques et militaires entre les pays occidentaux et les pays non-occidentaux, et à l'heure où la croissance démographique des premiers est beaucoup moins forte que celles des seconds, Huntington craint que les civilisations occidentales ne puissent assurer la suprématie mondiale. Il est conscient que la force est un élément décisif pour l"influence culturelle. Il prône le maintien par les pays occidentaux de forces économiques et militaires importantes pour sauvegarder leurs intérêts vitaux et conserver leur rôle principal dans l'équilibre mondial. Il affirme que "les civilisations universelles ne sont que l'aboutissement des forces universelles". Autrement dit, les civilisations universelles ne pourront se généraliser et le monde demeurer en paix que si l'Occident, avec à sa tête les Etats-Unis, maintient son hégémonie sur globe.

2 L'analyse des causes des conflits internationaux faite par Huntington a eu sans nul doute un retentissement. Cependant il reste un réaliste classique quand il explique les formes et la nature des conflits. Dans son esprit, ou le vent de l'Est l'emporte sur le vent de l'Ouest ou le vent de l'Ouest l'emporte sur le vent de l'Est, dans les conflits culturo- 
civilisationnels, tout comme les conflits de puissance et d'intérêts, sont une affaire d'affrontement sans réconciliation possible. Seules les forces économiques et militaires permettent de maintenir la suprématie. Quelques semaines avant la parution de "Clash of civilizations?", Huntington a publié un autre article polémique dans International security. Le thème du débat était le suivant: est-il nécessaire pour les Etats-Unis de maintenir leur primauté internationale ? La comparaison de ces deux articles permet de mieux comprendre les particularités théoriques de la pensée de Huntington. Dans son article intitulé "Why International Primacy Matters", il affirme qu'il n'y a aucun doute à avoir quant à l'importance pour les Etats-Unis de s'efforcer de maintenir leur suprématie internationale. "S'interroger sur l'importance de la suprématie, c'est s'interroger sur l'intérêt du pouvoir". Et la réponse à donner ne peut-être que positive : Bien sur cela est important dans la plupart des relations entre les hommes, même dans la famille, et il en est de même pour les affaires nationales et internationales. "Si les politistes n'étudiaient pas la puissance et la primauté, ils devraient se trouver une autre activité. Chaque pays, afin d'assurer la sécurité nationale, de protéger les intérêts de l'Etat et de créer un environnement international reposant sur son sens des valeurs et ses intérêts, s'efforce naturellement d'imposer sa suprématie aux autres. Ce n'est qu'en devançant tous leurs concurrents, et dans tous les domaines que les Etats-Unis sont en mesure de relever les défis que peuvent leur lancer les autres puissances. Selon Huntington depuis la fin de la guerre froide, le fondement de l'alliance occidentale est ébranlé ; bien que le sens des valeurs des Etats-Unis soit proche de celui des autres pays occidentaux, que des guerres les opposant soient inimaginables, l'antagonisme politique et économique ira en s'accentuant. De ce fait, il est normal qu'ils rivalisent pour détenir la suprématie.

3 Huntington réfute également les arguments avancés par certains économistes américains, qui croient que la compétition économique entre les pays occidentaux n'est pas un jeu à somme nulle (c'est à dire un combat où les pertes de l'une des parties équivalent aux gains de l'autre). Selon lui, les Etats-Unis ne devraient pas être si naïfs car, les dirigeants et les peuples des autres nations, n'ont pas cette conception des relations internationales. L'économie constitue le fondement le plus important de la puissance de l'Etat. Militairement, les Etats-Unis sont moins menacés depuis la fin de la guerre froide, il est donc plus important pour eux de maintenir une supériorité économique relative, y compris sur le Japon contrairement à ce que croient certains Américains. En effet, si le Japon dépasse les Etats-Unis dans le domaine des sciences et de la haute technologie, les Américains s'appuieront de plus en plus sur les Japonais pour obtenir de la technologie militaire. Or, si dans le futur les Etats-Unis et le Japon sont en désaccord sur des conflits militaires, les Japonais seraient capables de paralyser les Etats-Unis. Il cite divers exemples à ce propos, afin de prouver que le Japon s'efforce actuellement de remporter la guerre économique qui l'oppose aux Etats-Unis et que ces derniers perdront leur initiative politique s'ils sont perdent leur économique contre le Japon dans la guerre économique. Huntington conclut: les Etats-Unis constituent la force principale pour la défense de la paix mondiale, le porte-parole de la liberté, de la démocratie et de l'économie de marché ; si les Etats-Unis maintiennent leur place de première puissance économique et militaire, la stabilité, la prospérité et le sens des valeurs de la liberté seront garanties, car en dernière analyse, les intérêts américains représentent les intérêts de l'humanité2.

4 Ainsi, Huntington ne reconnait-il ni la possibilité du fusionnement des civilisations, ni l'élargissement de zones de coopération entre différents pays, ni la réduction des causes de 
conflits que des relations de dépendance mutuelle liées à une économie mondialisé pourraient engendrer. Examinons ce point de vue qui serait connu de nous : "les conflits entre les Etats et les groupes nationaux depuis la fin la guerre froide vont croissant. L'opposition entre les Etats-Unis et les autres grandes puissances perdurent. Cela est "naturel". Que ce soit entre les individus, entre les groupes ou entre les Etats, la fin d'une grande guerre ou des conflits jette les bases pour de nouveaux conflits"3. L'antagonisme, la lutte et les conflits entre les individus, les groupes et les nations sont perpétuels et absolus; l'union et la coopération sont temporaires et relatifs. Cela est "naturel". On comprend alors facilement pourquoi Huntington a affirmé que le paradigme qui domine les relations internationales est invariablement un paradigme construit autour du conflit.

Différentes conceptions du terme "politique" sont apparues depuis l'antiquité. Hitler a définit la "politique" comme : ...l'art pour une nation de la lutte à mort pour l'existence sur le globe" ${ }^{4}$. Le théoricien réaliste classique américain Hans J. Morgenthau affirmait : "La politique internationale est comme les autres politiques, une lutte pour le pouvoir" 5 . Huntington affirme lui aussi que la politique internationale n'est que conflits et lutte pour la suprématie entre Nations et groupes de Nations. Le point de vue "réaliste" de Huntington sur les relations humaines et la politique, comme sa "philosophie de la lutte" sont en Chine comme ailleurs, majoritaires, et ce depuis les temps les plus anciens. Sur la nature des parties en conflit dans cette lutte à mort (que ce soient des Etats, des nations, des classes, des idéologies, ou des civilisations), elle varie selon les auteurs.

6 Nous lisions récemment dans un journal chinois les phrases suivantes: "les relations d'intimidation entre les hommes n'ont virtuellement pas changé depuis les temps les plus anciens. ...En général, dans la société humaine, les relations entre les nations sont d'abord les relations d'intimidation: le grand intimide le petit et le riche intimide le pauvre. En outre, n'existe-t-il pas des différences entre les exploiteurs et les exploités, entre les dirigeants et les dirigés au sein d'un pays donné ? Dans les relations entre groupes ethniques l'un des groupes n'essaye-t-il d'asservir et d'exterminer l'autre?". N'est-il pas tout à fait naturel, pour les riches et les puissants, dans les relations humaines quotidiennes, d'intimider les pauvres et les impuissants ${ }^{6}$. Ceci est l'interprétation ordinaire de ce que l'on appelle la "politique du plus fort".

7 Si l'on observe de cette façon l'histoire et la société, on ne perçoit qu'un monde de lutte à mort perpétuelle entre des oppresseurs et des opprimés, entre des ennemis. Le soi-disant ordre stable, n'est que le résultat du triomphe d'un rival sur un autre ou de l'extermination d'une partie par une autre. La théorie du conflit des civilisations et celle de la suprématie internationale partagent toutes les deux le même objectif, mettre en garde le gouvernement et le peuple américains : les Etats-Unis doivent encore faire face à des ennemis importants, dans le domaine spirituel, politique, économique, et militaire. Dès lors il est peu surprenant de voir certaines critiques de Huntington déclarer que le point central de l'hypothèse de ce dernier était "le besoin pour les Etats-Unis d'un ennemi" sur qui triompher?

On peut dire incontestablement que là où il y a de la lutte pour le pouvoir, il y a de la politique. Mais, la coopération pour la défense des intérêts communs, le compromis pour apaiser les conflits, la coordination pour les actions unifiées sont-elles des actions politiques? Huntington ne fait aucun commentaire. 
$9 \quad$ La "politique" peut être également définit autrement: la politique est l'activité qui consiste à prendre une décision dans les intérêts d'une communauté et de la mettre en application"8. "la politique réside dans le processus de partage de la valeur sociale avec autorité". . Ce que l'on appelle ici, "activité" et "processus" peuvent être définis aussi bien comme conflit et lutte, que comme compromis et coopération. Si l'on comprend la politique de cette manière, la stabilité nationale peut se fonder sur la pluralité politique et démocratique, l'ordre et la libre concurrence peuvent coexister. Le conflit international peut constituer, à une époque et dans une région donnée, le problème majeur, comme à d'autres époques la coopération et la coordination peuvent dominer les échanges internationaux.

10 Si nous approfondissons cette question théorique, nous sommes confrontés à un sujet, qui a donné lieu ces dernières années, à des discussions acharnées au sein de la communauté des spécialistes de relations internationales aux Etats-Unis: les gains relatifs et les gains absolus. Les gains relatifs, font référence aux préoccupations de chaque nation pour son statut et sa force à l'échelle international comparativement aux nations, notamment ennemies. Même si deux nations retirent mutuellement de leur coopération internationale des gains mutuels, chacune d'entre elle préférera se battre pour obtenir "la plus grosse part du gâteau". Au cours de la guerre froide, la réduction des armements nucléaires américaines et soviétiques auraient dû être profitable aussi bien pour les Etats-Unis que pour l'Union soviétique, pourtant ils n'ont pas cessé de se quereller sur la question de la réduction leur arsenal respectif. La confrontation militaire ou la revendication territoriale sont des jeux à somme nulle, les gains de l'un sont les pertes de l'autre. Pour les défenseurs des gains relatifs, la compétition économique internationale est-elle aussi un jeu à somme nulle, car les forces économiques des pays ennemis peuvent se transformer en forces militaires. Sur la question de la sécurité nationale, on retrouve un point de vue traditionnel qui reprend la théorie de gains relatifs : dans la confrontation qui nous oppose à notre ennemi, la lutte est une question de vie ou de mort, où il ne faut pas espérer que l'ennemi pose de lui même son couteau. Pour préserver la paix, il est nécessaire, de bien faire la distinction entre nous et l'ennemi. La préparation de la guerre mobilise la nation toute entière, la maintient en alerte et développe la confiance et la cohésion nationales. Dans le domaine idéologique et culturel, il est crucial de maintenir les traditions nationales et de résister à la culture étrangère. En matière économique, les défenseurs des gains relatifs demandent une indépendance économique et la protection de l'industrie nationale. Ils craignent notamment qu'une pénétration étrangère dans l'économie nationale ne se traduisent par un transfert du capital national vers l'étranger. Ils présument donc une telle situation constituerait une menace pour l'indépendance politique de la nation.

11 La théorie des gains absolus a gagné en audience ces dernières années, avec la fin de la guerre froide. Les différentes nations commencent à se préoccuper à nouveau de leurs affaires intérieures, obsédées par le développement de l'économie nationale et le bienêtre social. Dans les relations économiques internationales, ce qui préoccupe le plus les défenseurs des gains absolus c'est le progrès effectué par le pays sans s'intéresser aux gains réalisés par les autres, par la coopération. Dans la mesure où les Etats ne sont pas au même niveau de développement, une fois les frontières respectives ouvertes, les pays plus développes posséderont un avantage certain. Cependant les pays, ou les secteurs de production archaïques pourront éventuellement améliorer leur 
compétitivité. Ceci est peut-être plus important que l'apparente "égalité internationale". Ils soutiennent que la coopération internationale devraient apporter des bénéfices mutuels. Puisque l'on veut attirer un investissement étranger, on ne devrait pas s'inquiéter outre mesure si plus d'argent finissent dans des poches étrangères.

12 La prolifération nucléaire, l'écologie, la contrebande, le trafic de drogue, l'exode de réfugiés, le terrorisme international, le sida, etc. constituent des problèmes communs à toute l'humanité. Aucun pays ne doit se réjouir de la perte enregistrée par un pays, même s'il s'agit d'Etats ennemis car les désastres des autres peuvent toucher éventuellement son propre pays. Nombreux sont ceux qui cherchent le sens de la valeur commune à l'humanité en vue de promouvoir la compréhension mutuelle et le fusionnement culturel sans considérer les valeur de leurs nations comme l'objectif ultime à atteindre. Concernant les questions militaires et de sécurité, les tenants de la théorie des gains absolus soulignent que la sécurité d'un pays dépend de la sécurité internationale. Selon eux l'éducation doit être fondée sur la paix et non sur la guerre ; il est nécessaire de développer des communications réciproques entre les nations ennemies potentielles, de réduire les frais militaires, d'apaiser les conflits frontalières, et d'enrayer le nationalisme extrême.

Bien sûr, la distinction entre la conception des gains relatifs et celle des gains absolus n'est pas aussi tranchée dans la vie politique pratique. Tout personne dotée d'un peu de bon sens politique, ne penserait ou n'agirait pas de façon aussi extrême. Néanmoins, les défenseurs des gains relatifs pourront facilement être considérés comme les "faucons", les partisans de la ligne dure, tandis que les défenseurs des gains absolus seront plutôt perçus comme des "colombes", des modérés, des "mondialistes". Huntington fait évidemment partie des premiers. Il ne fait que se d'une parure son nationaliste, lorsqu'il affirme que "l'intérêt américain est l'intérêt de l'humanité". Ses idées sur les relations internationales peuvent à n'en point douter être classés sous l'appellation de Robert Gilpin, "nationalisme économique", qui affirme que le but principal de l'activité économique de devrait pas être le bien-être social mais servir la construction nationale, la sécurité nationale et la suprématie internationale"10. La théorie du conflit des civilisations de Huntington reflète ses conceptions traditionnelles du pouvoir, son nationalisme étroit et son idéologie conservatrice sur la question des civilisations.

Christopher Layne et Robert Jervis, deux adversaires de Samuel Huntington, montrent dans "International Security" qu'au moment où la compétition économique remplace de manière évidente la confrontation militaire, devenant une donnée majeure des relations internationales et dans des conditions telles que les heurts entre les EtatsUnis et les autres pays développés ne conduiront pas à la guerre, Il n'est pas nécessaire pour les Etats-Unis de se lancer dans des épreuves forces avec les autres nations développées pour maintenir leur suprématie. Exemple typique d'idée relevant de la théorie des gains absolus telle peut être présentée par des spécialistes américains des relations internationales. Ces deux spécialistes estiment que les Etats-Unis devraient se satisfaire de l'apparition d'un monde multipolaire, s'efforcer de jouer dans cette situation le rôle de point d'équilibre stratégique du globe, celui de la puissance hégémonique prenant à sa charge la stratégie globale ; la ressaisissement économique américain doit être fondée sur la prospérité économique des autres industrialisés ; considérer la prospérité de ces derniers comme menace pour les Etats-Unis est sans fondement ${ }^{11}$. 
facilement comment les spécialistes américains qui soulignent la dépendance mutuelle (transnationalisme) et défendent la globalisation réagissent à la théorie de Huntington. Un débat intense a opposé pendant environ deux décennies, au sein de la communauté des spécialistes américains, les défenseurs du néo-réalisme (réalisme structurel) et de la dépendance mutuelle à ceux $\mathrm{du}$ réalisme traditionnel. Les premiers occupent aujourd'hui une position dominante. Les transnationalistes montrent que le commerce, le crédit, l'investissement, l'aide étrangère, l'écologie et les autres questions de "basse politique" (low politics) deviennent de plus en plus les questions centrales des relations internationales et des politiques étrangères. Les réalistes, quant à eux maintiennent que les questions relatives à la sécurité nationale, au prestige, à la puissance, à la souveraineté, au territoire et les autres questions de "haute politique (high politics) demeurent encore primordiales. Samuel Huntington fait des chocs entre les civilisations une dernière ligne de défense de la haute politique. Ainsi peut-on expliquer en partie pourquoi sa nouvelle théorie n'a pas provoqué de réaction majeure aux Etats-Unis ou dans le reste des pays occidentaux. A l'inverse, les ondes de choc qu'elle a provoquées en Chine et dans d'autres pays non-occidentaux montrent qu'il y a bien des psychologies nationales, des idées politiques et des intérêts académiques divergents entre les nations de niveaux de développement et de statuts internationaux différents. traditionnelle, la volonté de comprendre, critiquer et absorber la culture occidentale, l'attitude politique pragmatique, les prédictions et analyses de l'avenir politique de la Chine et les désirs d'améliorer son statut international après le renforcement de sa puissance économique, se sont tous combinés pour créer une ambiance politicoculturelle unique. Ainsi, l'intérêt unanime de la communauté académique chinoise pour l'article de Huntington s'apparente plutôt à une reformulation théorique qu'à une réponse. Cette reformulation théorique devrait revenir sur la question de la nature de la politique internationale, si elle est par essence une lutte entre les Etats pour la conquête de territoire, des ressources, une lutte entre des intérêts économiques, une lutte pour la suprématie.

\section{LA SIGNIFICATION POLITIQUE PRATIQUE}

17 La prévision faite par Huntington, conseiller du prince, dans son analyse du conflit de civilisations peut être résumée en trois phrases : la civilisation sera l'élément distinctif principal dans la division politique du monde futur ; des conflits apparaitront entre les pays occidentaux et les pays non-occidentaux ; les gouvernements occidentaux doivent se méfier du monde islamique et surveiller l'éventualité d'une alliance confucianoislamique. Et Huntington de préciser que la deuxième proposition demeure essentielle.

Même si les spécialistes des relations internationales, et ceux qui sont chargés aujourd'hui, dans les principaux pays du monde, de définir la politique diplomatique peuvent ne pas tenir compte des prédictions faites par Huntington, ils doivent cependant évaluer les questions d'importance stratégique qui se poseront à long terme à savoir : le monde de l'après de la guerre froide se caractérise-t-il par les conflits ou l'apaisement, par le désordre ou la stabilité, par la division ou l'unité ? Quelle sera la structure future des relations internationales, autrement dit, quels seront les principaux acteurs politiques? Quelles seront leurs relations? Où éclateront les principaux conflits? 

donné semble-t-il, malgré les nombreuses réfutations de sa théorie, une réponse plus claire et plus complète que lui. Sans doute est-ce l'une des raisons pour laquelle Huntington demeure satisfait, malgré les violentes critiques dont il a été l'objet.

ne cherchons pas ici à présenter une théorie structurelle, prédictive, alternative au paradigme du conflit des civilisations. Sommes-nous d'ailleurs capables, en tant que scientifiques, de formuler autre chose qu'un jugement fondé sur l'intuition et la représentation, c'est à dire des hypothèses discutés et démontrées dans les milieux académiques, sur ce problème majeur, mais abstrait? Si la réponse est négative, nous devrions le laisser de côté, et nous intéresser à des problèmes de moindre envergure. Par exemple : quels sont les facteurs qui provoqueront de nouveaux conflits, du désordre et de la division? Quels facteurs encourageront la stabilité, l'intégration, le calme?

21 Il n'est pas nécessaire de formuler ici des jugements sur la valeur positive ou négative de ces facteurs? Il faut garder à l'esprit que tout intégration partielle (comme les groupements économiques) conduirait probablement à une division du monde ${ }^{12}$. Pendant la Révolution Culturelle en Chine, on disait: " Il y a un désordre général, la situation est excellente ". C'était un jugement de valeur correspondant à une époque particulière, avec ses raisons particulières reflétant l'état d'esprit du moment. Même aujourd'hui, tout le monde ne croit pas que le calme, la stabilité, l'interdépendance et la globalisation favorisent ses intérêts particuliers même si peu de discours s'oppose à cette tendance. La théorie de Huntington permet de comprendre que non seulement il ne croit pas à la possibilité d'un fusionnement ni d'une coopération dans long terme entre l'Occident et le non-Occident, mais il ne les considère pas comme souhaitables. Si les choses évoluent comme Huntington le prédit, plus l'interdépendance s'accentuera, plus l'intégration s'accélérera, plus le choc ressentir par la civilisation occidentale, telle qu'elle est défendue par Huntington et ses semblables, sera violent. Eprouvant un sentiment, l'Occident manifestera le désir de plus en plus marqué de prendre des mesures, y compris des actions prévoyant le recours à la force armée, pour résister à une telle tendance vers la globalisation.

Concernant la question des relations entre la démocratie politique, les droits de l'homme et la souveraineté, Huntington se soucie principalement de l'alliance, tant visible qu'invisible, des pays en développement contre l'Occident. Il craint que ce dernier ne puisse maintenir sa suprématie politique. Pour nombre de pays en développement comme la Chine, l'Inde, le Pakistan, l'Irak, l'Iran, l'Indonésie, la Malaisie, Singapour, Cuba, la Corée, le Vietnam, la Birmanie, le Zaïre, la Libye, le Soudan, la résistance à la pression politique de l'Occident constitue, si ce n'est l'objectif principal de leur diplomatie, du moins un objectif important. l'Iran a proposé publiquement la constitution d'un front uni contre les Etats-Unis. Concernant la question de la non prolifération nucléaire et des armes de haute technologie, l'opinion et les intérêts de l'Occident et du monde non-occidental sont à ce point différents que les Etats-Unis se croient aujourd'hui, et pour longtemps, menacés. Dans le domaine économique, l'antagonisme entre le Sud et le Nord est également celui de l'Occident et du non-Occident. La population du monde non-occidental émigre en Europe et, de plus en plus d'Asiatiques et de Sud-Américains vont s'installer aux Etats-Unis. Ceci réduit la proportion de la population occidentale dans la population totale mondiale, et ajoute à ses conflits internes, rendant plus complexes les problèmes sociaux intérieurs. Samuel Huntington ne se trompe pas semble-t-il lorsqu'il se réfère au défi posé au monde 
occidental par le monde non-occidental. Mais la véritable question est de savoir si cela va déboucher sur un choc des civilisations comme il le prétend, et si les oppositions entre l'Occident et le monde non-occidental seront supérieures aux conflits "internes". Le célèbre futurologue américain Alvin Toffler a proposé lui aussi une version "du choc des civilisations" dans un ouvrage paru en 1993. Il a essayé de démontrer que la "troisième" vague" de la révolution scientifique et technologique a créé une nouvelle civilisation constituée par les pays en possession des nouveaux moyens d'information. L'opposition entre la civilisation formée des pays occidentaux avancés d'une part et les civilisations industrielles des pays non-occidentaux fondées sur un travail intensif et les civilisations agricoles archaïques d'autre part pourra déboucher sur une guerre générale de typesofware contre acier ${ }^{13}$. Bien que la définition de la civilisation de Toffler soit complètement différente de celle de Huntington, bien que leurs moyens et leurs arguments diffèrent, ils se rejoignent toutefois sur la prédiction de chocs de civilisations.

Bien que les affrontements économiques entre Etats occidentaux se multiplient, les observateurs politiques mondiaux s'accordent pour dire que de telles confrontations économiques ne se transformeront pas en confrontations politiques ou en conflits armés. Il est plus probable de voir éclater de graves conflits armés entre des pays en développement où se mêlent des litiges territoriaux, conflits nationaux, lutte entre les groupes religieux et conflits d'intérêts économiques. Exemple : rares sont ceux qui peuvent affirmer qu'il n'y aura pas de guerre majeure entre l'Inde et le Pakistan. Par ailleurs, il faut prêter une grande attention à l'orientation politique futur du Japon et de la Russie, lesquels ne font pas partie à la civilisation occidentale chrétienne. Samuel Huntington s'intéresse peu au Japon dans son article "The Clash of civilizations", se contentant de le classer parmi les civilisations non-occidentales.

Cependant, dans son article "Why International Primacy Matters", il montre de l'anxiété et de méfiance à l'égard de ce pays qu'il considère comme un terrible rival des Etats-Unis. En feuilletant des publications de relations internationales parues ces dernières années, nous remarquons facilement que les Américains s'accommodent plus aisément des défis politiques et économiques lancés par les autres pays développés que ceux que lui lancent le Japon. Les significations politiques d'un tel constat sont pour le moins ambiguës. Un rapport de la Rand Corporation de 1993 sur les relations nippoaméricaines affirme qu'après la disparition de l'Union Soviétique, les liens entre le Japon et les Etats-Unis se fondent sur une perception des valeurs et un système politique communs; si le Japon tente de se s'éloigner des Etats-Unis et ne se reconnaît plus dans les valeurs occidentales de liberté et démocratie, les relations de confiance entre les deux pays seront menacées. Ce problème n'intéresse pas seulement les spécialistes et les politistes, mais également les responsables politiques américains. Le rapport poursuit: "actuellement, de nombreux nationalistes japonais pensent que le Japon peut être, pour les autres pays asiatiques et même pour le monde entier, un modèle particulier non-occidental de développement social et économique. Cette conviction suscite une sympathie dans certains pays asiatiques". Les nationalistes critiquent également violemment la société américaine en dénonçant l'hypocrisie de sa diplomatie des droits de l'homme alors que les USA connaissent de graves conflits raciaux. Ces nationalistes se rendent compte également que "l'avantage particulier du Japon ne réside pas dans une plus grande ouverture mais dans son homogénéité culturelle et raciale. Cette caractéristique permet au Japon de parvenir à parvenir un objectif social collectif impossible à réaliser au sein la société multi-ethnique 
américaine ${ }^{14}$. La Chine a enregistré ces dernières années un accroissement de ses forces économiques et de son influence politique, la "théorie de la menace chinoise" est apparu au même moment dans les milieux politiques américain. Ce n'est tant la force croissante de la Chine qui inquiète mais la réussite éventuelles du socialisme à la chinoise qui constituera un défi au sens des valeur de l'Occident. Dans l'esprit de Huntington et d'autres conseillers américains il est improbable que le Japon et d'autres pays asiatiques parviennent à se "moderniser sans être occidentalisés", mais cette option constitue une variable importante que les responsables politiques américains ne pourront ignorer dans l'élaboration de leur stratégie sur le long terme. Dans son "Clash of Civilizations", Samuel Huntington avance l'idée que l'axe central des relations internationales dans le futur sera probablement constitué par le conflit entre le monde occidental et le monde non occidental, sur la réaction des civilisations non-occidentales contre le pouvoir et le sens de valeur de l'Occident; un des choix qui s'offre aux pays non-occidentaux consiste à accroître leurs forces économiques et militaires et de coopérer contre l'Occident tout en protégeant leur sens des valeurs et leurs institutions politiques propres ${ }^{15}$.

Les défis lancés aux valeurs occidentales traditionnelles ne proviennent pas nécessairement des pays non-occidentaux. Dans ses articles qui répondent aux critiques qui lui ont été adressées Huntington, laisse transparaitre enfin ses préoccupations les plus profondes; en 2050, il y aura aux Etats-Unis 23\% d'Hispaniques, $16 \%$ de Noirs et $10 \%$ d'Américains-Asiatiques, c'est à dire que presque la moitié de la population américaine ne sera pas blanche. Il demande donc: ces nouveaux émigrants seront-ils assimilés dans la culture européenne jusqu'à maintenant prédominante aux Etats-Unis? ". Il demande "Les nouveaux immigrants seront-ils intégrés à la culture européenne, dominante jusqu'à alors, des Etats-Unis?". Si les Etats-Unis continuent leur politique d'immigration actuelle, multiculturelle et multiraciale, un choc des civilisations éclatera à l'intérieur du pays, les Etats-Unis, tels que nous les connaissons disparaîtront et ils suivront l'Union Soviétique dans la poubelle de l'Histoire ${ }^{16}$.

Huntington dit ici, ce que beaucoup d'Américains blancs (WASP) n'osent pas dire.La critique de la culture occidentale s'est faite plus en plus vive ces dernières années au sein de la société américaine. Certaines initiatives comme celles permettant aux femmes et aux minorités nationales d'avoir accès plus facilement à l'éducation ou à un travail, ou certains aspects de la vie sociale comme la plus grande tolérance manifestée à l'égard de l'homosexualité, sont considérés par nombre d'américains blancs, conservateurs, comme excessifs ${ }^{17}$. Cette évolution s'est traduite par l'idée du "politiquement correct". Nombre d'Américains considèrent néanmoins que l'inquiétude de Huntington face à la pluralité raciale et culturelle aux Etats-Unis n'est pas "politiquement correcte". Il existe une autre tendance que Huntington oublie de mentionner, c'est la combinaison entre le défi extérieur lancé par le monde non-occidental et le mouvement contre-culturel américain. L'antagonisme entre le monde occidental et non-occidental pourrait exacerber les conflits raciaux aux Etats-Unis, en outre l'accroissement de l'influence politique des groupes raciaux non-blancs et leurs liens multiples avec les forces étrangères perturberont probablement la réalisation de l'objectif de fixé par la diplomatie américaine. $\mathrm{Vu}$ sous cet angle, la politique intérieure américaine et les changements sociaux dans le pays auront une influence insoupçonnable sur la stratégie de la diplomatie américaine. 
Les approches conservatrices de Huntington à l'égard des questions d'ordre interne aux USA, ainsi que sa perspective de gains relatifs en ce qui concerne les questions internationales forment un système de représentation complet. On peut facilement imaginer que les idées politiques de Huntington ont rencontré une forte opposition dans les milieux politiques et académiques américains. Par conséquent, il serait exagéré de le considérer comme porte-parole officiel des Etats-Unis et de croire que sa théorie du conflit des civilisations pourrait constituer le fondement de la stratégie future de la diplomatie américaine. Néanmoins, il ne fait aucun doute qu'il a exprimé un sentiment profond partagé par certains sommets de la hiérarchie politique américaine et représente le retour de l'idée "la négation de la négation".

Nous ne devons pas surestimer le rôle de la théorie de Huntington dans l'établissement de la politique diplomatique américaine, mais il ne faudrait pas non plus le sousestimer si nous considérons cette théorie comme la manifestation d'un état d'esprit général, d'un jugement de valeur et d'un choix de politique. Comme le dit justement Karl R.Popper : la prévision peut influencer ce qui est prévu $^{18}$. Huntington rationalise l'idée de suprématie des nations; il considère comme dangereuse toute forme développement qui ne s'inscrirait pas dans le sens des valeurs occidentales traditionnelles (les valeurs de la civilisation blanche et chrétienne); il perçoit tout pays capable de concurrencer les Etats-Unis comme un ennemi potentiel ; il décrit la société future comme un monde dominé par les conflits. Si la pensée politique de Huntington se réalise, sa prédiction produira l'effet d'Oedipe" et deviendra une "prophétie auto-réalisatrice" : si vous pressentez qu'un pays sera votre ennemi, vous vous méfiez de lui, vous essayez de l'isoler, et vous l'attaquez. Par ce mécanisme, un pays, qui n'était pas un ennemi, devient méfiant et essayera de vous attaquer à son tour. Votre prophétie agit de telle sorte qu'elle favorise la confrontation, et devient malheureusement la réalité.Pour critiquer la conception des civilisations et de la théorie du conflit de Huntington, et empêcher que sa prophétie ne devienne réalité, il est nécessaire de ne pas retomber dans les mêmes errements. Si l'on veut sincèrement voir la paix et la prospérité dominer le monde, il ne faut pas souhaiter comme le fait Huntington que son pays, sa race, soit l'entité suprême, dominant tous les autres. Si l'on veut observer le développement de la civilisation mondiale, sans étroitesse d'esprit, on ne doit pas affirmer que seule la philosophie de sa nation pourra sauver l'humanité, ni prétendre à la supériorité de sa civilisation sur toutes les autres; ni croire que l'intelligence de sa nation et de sa race est mieux développée que celle des autres nations et autres races, ni même que sa langue et son écriture sont plus belles et plus raffinées que les autres, afin de provoquer une sensation, fausse, de supériorité et de renforcer la "cohésion nationale" exclusive. Les véritables penseurs immortels de l'histoire humaine, comme Confucius, Platon, Kant, Marx n'appartiennent pas seulement à une civilisation ou à une nation, mais à l'humanitéentière. Bien qu'ils aient vécu dans un environnement historique et géographique différent du nôtre, vu un monde différent, ils ont désiré construire une civilisation universelle. Quand Samuel Huntington déclare que "la civilisation universelle ne peut être que le produit du pouvoir universel", peut-être est-ce vrai. Mais, inversement, le pouvoir universel ne pourra être créé que si les civilisations fusionnent, et non si elles combattent. Un tel monde est encore loin de nous, mais il mérite que l'on y travaille avec détermination pour qu'il existe. 


\section{NOTES}

1. . Samuel P. Huntington, "The U. S., Decline or Renewal ?", Foreign Affairs,Vol.67, $\mathrm{n}^{\circ}$ 2, Winter 1988-89, p. 76-96.

2. . Huntington (S.P.), "Why International Primacy Matters", International Security, Vol. 17, No. 4, Spring 1993, p. 68-83.

3. . Samuel P. Huntington, "Why International Primacy Matters ?", op.cit., p. 71.

4. . Roger Scruton, A Dictionary of Political Thought, New York, Hilland Wang, 1982, p. 361.

5. . Hans J. Morgenthau, Politics Among Nations : The Struggle for Power and Peace, New York, Alfred A.Knopf, 1985, p. 31.

6. . Li Jiefei, "The Same Kind Bullies Each Other", South Weekend Paper, 19 novembre 1993, 6e édition.

7. . Lin Su, ibid., p. 25.

8. . David Miller et Bogdanov, Encyclopédie de Politologie Bolchevik, Edition de

l'Université des Sciences politiques et juridiques de Chine, 1992, p. 583.

9. . David Easton, The Political System, New York, Knopf, 1959, p. 129-131.

10. . Robert Gilpin, The Political Economy of International Relations, Princeton, N. J., Princeton University Press, 1987, p. 31-34.

11. . Christopher Layne, "The Unipolar Illusion : Why New Great Powers Will Rise", and Jervis, op. cit., International Security,Vol.17,No 4, Spring 1993, p. 5-67. A propos de la présentation et de la critique du débat, voir mon article "Why International Primacy is Necessary Comments on a Debate among the american Community of Scholars of International Politics", in The Orient, $\mathrm{n}^{\circ}$ 2, 1993.

12. . A propos des relations dialectiques de convergence et de division du monde après la guerre froide, voir John Lewis Gaddis, The United States and the End of the Cold War : Implications, Reconsiderations, Provocations, Oxford University Press, 1992, Chapter II.

13. . Alvin Toffler, Heidi Toffler, War and Anti-War : Survival at the Dawn of the 21st Century, Boston, Little, Brown and Company, 1993.

14. . Francis Fukuyama, Kongdan Oh, The U. S. - Japan Security Relationship After the Cold War, Rand National Defense Research Institute, 1993, p. 35-40.

15. . Samuel P. Huntington, "The Clash of Civilizations", op.cit.,p. 41.

16. . Samuel P. Huntington,"If Not Civilizations, then What ?", op.cit., p 190.

17. . En ce qui concerne le défi contre le courant principal de la culture américaine, voir Shen Zongmei, "Le défi contre le courant principal de la culture américaine", Les Etudes sur les Etats-Unis, n 3, 1992, p. 118-149.

18. . Karl R.Popper, The Poverty of Historicism, London, Routledge \& Kegan Paul, 1979, p. 13. 
INDEX

Mots-clés : menace, identité, Relations Internationales

Index géographique : Chine 